\title{
A prospective study of the radiological changes in the cervical spine in early rheumatoid disease
}

\author{
J. WINFIELD, D. COOKE, ${ }^{1}$ A. S. BROOK, ${ }^{2}$ AND MARY CORBETT
}

From the Department of Rheumatology, Middlesex Hospital, Arthur Stanley House, Tottenham Street, London WIP 9PG

SUMMARY The cervical spine radiographs of 100 patients with early rheumatoid disease were studied annually, on a prospective basis, for a mean follow-up period of 7 years 2 months. Atlantoaxial subluxation developed in 12 patients. The subluxation was more frequent in females, more severe in patients with progressive, seropositive, erosive rheumatoid disease, and more marked in patients treated with oral corticosteroids. Subaxial subluxation, affecting upper cervical disc levels, occurred in a further 20 patients. Three patients developed vertical subluxation. The mobility of the cervical spine affects the degree of subluxation achieved, and when assessing serial films for subluxation it may be necessary to measure the cervical spine flexion before deciding whether subluxation has progressed or not. Over $80 \%$ of the patients with subluxation developed the first evidence of subluxation within 2 years of disease onset. Subluxation in the cervical spine is not, therefore, a late complication of rheumatoid disease. During the follow-up period none of the patients developed neurological signs.

Cervical spine involvement in rheumatoid arthritis is common. Sharp ${ }^{1}$ found that the cervical spine was affected clinically at some stage of the disease in $40 \%$ of the patients with rheumatoid arthritis attending the Rheumatism Research Centre in Manchester. Bland et al. ${ }^{2}$ found radiological evidence of cervical spine involvement in $86 \%$ of cases of classical or definite rheumatoid arthritis. In population studies Lawrence ${ }^{3}$ found the prevalence of radiological changes of rheumatoid arthritis of the cervical spine to be $4.1 \%$ in males and $4.7 \%$ in females aged 15 and over, and the prevalence increased with age to around $15 \%$ in patients aged 65 years and over.

There have been no previous prospective studies of the radiological changes which affect the cervical spine in early rheumatoid arthritis, and little is known about the development and progression of these changes. In the older patient it may be impossible, clinically and radiologically, to differentiate the effects of rheumatoid involvement

\section{Accepted for publication 25 March 1980}

${ }^{1}$ Present address: Department of Medicine and Rheumatology, Merlin Park Regional Hospital, Galway, Ireland.

${ }^{2}$ Present address: Woden Valley Hospital, PO Box 11, Woden, ACT 2606, Australia.

Correspondence to Dr J. Winfield, Arthur Stanley House, Tottenham Street, London W1P 9PG. of the cervical spine from superimposed degenerative changes.

This prospective study was undertaken to document more precisely the development and progression of the radiological changes of cervical spine involvement in early rheumatoid disease.

\section{Patients and methods}

In 1966 the Department of Rheumatology at the Middlesex Hospital began a prospective study of patients with early rheumatoid disease. The organisation of this study has been previously described. ${ }^{4}$ Patients were invited to enter the study if the rheumatologist suspected rheumatoid disease of less than 1 year's duration. This has enabled us to study clinically and radiologically the early features, natural history, and course of the disease almost from onset.

As part of this study cervical spine radiographs of 100 patients with early rheumatoid disease were taken annually. There were 44 men and 56 women. The mean age at entry to the study was $47 \cdot 6$ years. At entry $64 \%$ of the patients had 'definite' or 'classical' rheumatoid disease and $36 \%$ had 'possible' or 'probable' rheumatoid disease, ${ }^{5}$ and $42 \%$ were SCAT (sheep cell agglutination test) positive at 
presentation. The mean duration of follow-up was $7 \cdot 2$ years.

Plain lateral radiographs of the cervical spine were taken in full flexion and full extension using a $5 \mathrm{ft}(1.5 \mathrm{~m})$ tube-to-plate distance with the beam centred on the second vertebra. Patients were asked to flex and extend their necks until discomfort or stiffness inhibited further movement. The 7 radiological criteria used in this study for the diagnosis of rheumatoid arthritis of the cervical spine are set out in Fig. 1. The criteria are modified from the study of Bland. 6 The radiographs were read by 2 of the authors (J.W. and D.C.) without knowledge of the clinical or serological data.

Atlanto-axial subluxation was measured by recording the shortest distance between the posterior surface of the anterior arch of the atlas to the anterior surface of the odontoid peg. A distance of $2.5 \mathrm{~mm}$ or more was taken as significant.

Subaxial subluxation was recorded as present if displacement between adjacent vertebral bodies was more than $1 \mathrm{~mm}$ or more than $15 \%$ of the anteroposterior vertebral diameter using the grading of Smith et al. ${ }^{7}$ Vertical subluxation was recorded as present if the tip of the odontoid peg lay more than $4.5 \mathrm{~mm}$ above the line described by McGregor. ${ }^{8}$

Disc space narrowing at $\mathrm{C} 2-3, \mathrm{C} 3-4$, and $\mathrm{C} 4-5$ was recorded only if there was a relative lack of osteophytosis. Disc space narrowing at lower levels in the cervical spine was not documented, since degenerative changes are frequently superimposed.

Vertebral plate erosions, often accompanied by localised areas of reactive sclerosis, were documented.
Apophyseal joint erosions and sclerosis were also looked for. The obliquity of the apophyseal joints, however, frequently obscured the definition of these joints on a plain lateral film, making erosions difficult to visualise. Osteoporosis was assessed subjectively as present or absent.

As the degree of subluxation in the cervical spine usually varies with the degree of neck flexion, the cervical spine flexion was measured by recording the distance in centimetres between the most posterior and inferior point of the spinous process of $\mathrm{Cl}$ to a similar point on the spinous process of C7. In addition the vertical height of the cervical spine was recorded to document any reduction in height of the cervical spine which may occur with time. The distance in centimetres from the centre of the anterior arch of the atlas to the centre of the body of $\mathrm{C7}$ was recorded.

\section{Results}

\section{ATLANTO-AXIAL SUBLUXATION}

Twelve patients developed atlanto-axial subluxation 9 females and 3 males. Ten of these patients had seropositive, erosive rheumatoid disease treated with gold or D pencillamine. Despite this therapy 7 of the 10 patients showed an increase in atlanto-axial subluxation (AAS) over a mean follow-up period of 52 months, detectable even though the cervical spine flexion had decreased in serial films in all 7 cases. In 3 patients with seropositive, erosive disease the AAS decreased without the development of vertical subluxation or marked odontoid erosion,

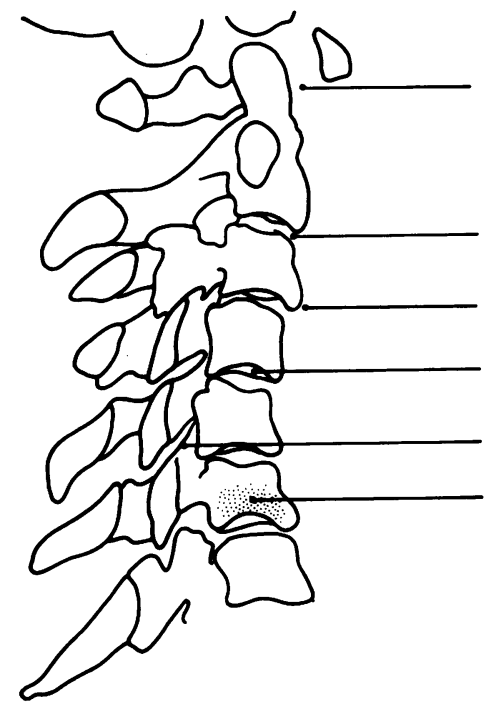

Atlanto-axial subluxation of $2.5 \mathrm{~mm}$ or more

Vertical subluxation

Disc space narrowing affecting upper cervical discs without osteophytosis

Multiple subluxation of $1 \mathrm{~mm}$ or more

Vertebral plate erosions and sclerosis

Apophyseal joint erosions and sclerosis

Fig. 1 Radiographic criteria for rheumatoid arthritis of the cervical spine.

Osteoporosis 
but in each case the degree of cervical spine flexion decreased with time, thereby reducing the degree of subluxation. Two patients were persistently seronegative and nonerosive as far as their peripheral joints were concerned, but each showed a steady increase in AAS during their mean follow-up period of 7 years 10 months. One such patient had AAS increasing from 6 to $8 \mathrm{~mm}$ over 8 years before the development of vertical subluxation. The other patient developed progressive AAS from $2.5 \mathrm{~mm}$ to $4 \mathrm{~mm}$ over 3 years, after which the subluxation remained static for a further 3 years.

\section{TIMING OF THE SUBLUXATION}

At their first visit to the hospital, that is, within 1 year of disease presentation, 4 of the 12 patients already had evidence of AAS. In 3 of these the AAS remained static and did not deteriorate during the follow-up period, but in the fourth a progressive deterioration was seen before the development of vertical subluxation. It is impossible to say when subluxation first developed in these 4 patients in relation to the onset of their rheumatoid disease.

A further 6 patients had evidence of AAS within 2 years of disease presentation, and, of these, 5 continued to have seropositive, erosive rheumatoid disease frequently difficult to control, and the degree of AAS steadily increased, while in 1 patient the AAS was static.

Of the remaining 2 patients in the subluxation group both showed a series of progressive and static phases in the development and progression of subluxation with AAS occurring after 3 and 4 years respectively.

This indicates that the majority of patients $(83 \%)$ with subluxation showed the first evidence of the subluxation within 2 years of disease presentation.

All patients in the AAS group showed other radiological changes of rheumatoid disease in their cervical spines. The associated changes were, in order of frequency (i) marked apophyseal and vertebral plate sclerosis (8); (ii) disc space narrowing involving $\mathrm{C} 2-3, \mathrm{C} 3-4$, and $\mathrm{C} 4-5$ (7); (iii) multiple level subaxial subluxation (4); (iv) irregularity and probable erosion of the odontoid peg giving a pointed appearance (4); (v) fusion of the C2-3 apophyseal joints (2).

Three of the 12 patients with AAS had received regular oral corticosteroids, and the subluxation was more marked in this group, the mean AAS being 6 $\mathrm{mm}$ compared with the remainder of the group who had never received corticosteroid treatment and had a mean AAS of $4.5 \mathrm{~mm}$. Comparison of the duration of disease, overall disease state before treatment, therapy, and functional grade between the steroid treated and nonsteroid treated patients did not indicate any significant differences. Patients receiving corticosteroids were not able to achieve any greater degrees of mobility of the cervical spine as a whole than patients who did not receive corticosteroids, and in both groups the cervical spine flexion decreased with time. Subaxial changes were similar in the steroid and nonsteroid treated groups, suggesting that corticosteroids do exert a deleterious effect on the atlanto-axial joint in particular. The development of AAS with softening, laxity, and often rupture of the transverse and alar ligaments, combined with osteoporosis of adjacent bone, is probably exacerbated by corticosteroids.

The most common symptoms in the AAS group were cervical spine pain and stiffness (6 patients). One patient developed severe occipital and frontal headaches, probably due to irritation of the $\mathrm{C} 2$ root. No patient, however, developed abnormal neurological signs during the follow-up period.

\section{SUBAXIAL SUBLUXATION}

Subaxial subluxation occurred in 24 patients in all; 4 of these also had atlanto-axial subluxation. In 16 patients the degree of subluxation was mild, producing only $1 \mathrm{~mm}$ displacement between adjacent vertebral bodies. This occurred at a single level in six patients $\left(\mathrm{C}_{2}-3 ; 2 ; \mathrm{C} 3-4: 4\right)$, at multiple levels $(\mathrm{C} 2-3, \mathrm{C} 3-4$ and $\mathrm{C} 4-5)$ in 8 patients, and in the remaining 2 ankylosis of the apophyseal joints and bony fusion of the vertebral bodies of $\mathrm{C} 2$ and 3 was found. Five patients with subaxial subluxation affecting $\mathrm{C} 2-3, \mathrm{C} 3-4, \mathrm{C} 4-5$ with more than $2 \mathrm{~mm}$ displacement at each level showed a series of progressive and static phases in the development of the subluxation in their serial radiographs over a mean follow-up period of 8 years. The remaining 3 patients in the subaxial subluxation group showed gradual progression of subluxation at $\mathrm{C} 2-3, \mathrm{C} 3-4$, $\mathrm{C4}-5$, rather than a series of progressive and static phases.

Overall, the subaxial subluxation was detectable at presentation in 12 patients, within 2 years in 7 patients, within 4 years in 2 patients, and within 5 years in the remaining 3 patients. None of the patients developed abnormal neurological signs. The other associated radiological changes with subaxial subluxation were multiple-level disc space narrowing in 18 , vertebral and plate erosion and sclerosis in 9, marked osteoporosis in 10, and marked apophyseal joint sclerosis in 6 .

\section{VERTICAL SUBLUXATION}

Vertical subluxation was found in 3 patients and ranged between $5.5 \mathrm{~mm}$ and $10 \mathrm{~mm}$ above McGregor's line. Two of the patients had AAS which was detectable before vertical subluxation occurred. 
In one patient the AAS increased from 3 to $4 \mathrm{~mm}$ over a 5-year period and then apparently showed a decrease to $3 \mathrm{~mm}$ over a further 3-year period. During this time, however, vertical subluxation with upward migration of the odontoid peg could be seen on serial films. The mechanism of this decrease in atlanto-axial subluxation has been explained by Mathews. ${ }^{9}$ The vertical subluxation increased to $10 \mathrm{~mm}$ above McGregor's line. The other patient with AAS and vertical subluxation showed a similar sequence. The remaining patient in this group did not have evidence of peripheral erosive disease and AAS could not be demonstrated on the radiographs, and yet a steady upward migration of the odontoid peg from $5.5 \mathrm{~mm}$ to $9 \mathrm{~mm}$ over a 5 -year period could be seen. It is likely that AAS and vertical subluxation occurred at the same rate in this patient.

CERVICAL SPINE FLEXION AND SUBLUXATION Of the 33 patients with all forms of subluxation 19 patients showed a decrease in cervical spine flexion with respect to time. The most marked decrease in neck flexion was found in patients with AAS with a mean decrease of $1.8 \mathrm{~cm}$ over the followup period. Despite the reduction in cervical spine flexion an increase in atlanto-axial subluxation could still be recorded in 7 patients. The remaining 12 patients with subluxation and a decrease in neck flexion had mild subaxial changes only and principally complained of pain and stiffness of the cervical spine. In 5 patients the cervical spine flexion was static during the follow-up period. An increase in cervical spine flexion was found in 9 patients, the mean increase being $1.1 \mathrm{~cm}$ in the follow-up period. These patients had principally subaxial changes, especially at $\mathrm{C} 2-3$ and $\mathrm{C} 3-4$. Only 1 patient in this group had AAS. Mobility of the cervical spine, maintained while subluxation is developing, may encourage the development of subaxial subluxation, perhaps by preventing ankylosis at the apophyseal joints. Indeed the degree of sclerosis at the apophyseal joints in this latter group was subjectively less than in the group where a decrease in cervical spine flexion was found.

Only 6 patients in the subluxation group showed a decrease in cervical spine height over the follow-up period (mean decrease $0.6 \mathrm{~cm}$ over follow-up period of 7 years 8 months), and in all these there was a decrease in cervical spine flexion in serial radiographs. The decrease in flexion may therefore have been due in part to a decrease in height of the cervical spine due to disc space narrowing and loss of vertebral body height.

The majority of patients with subluxation showed several other radiological changes of rheumatoid
Table 1 Radiological abnormalities with and without subluxation

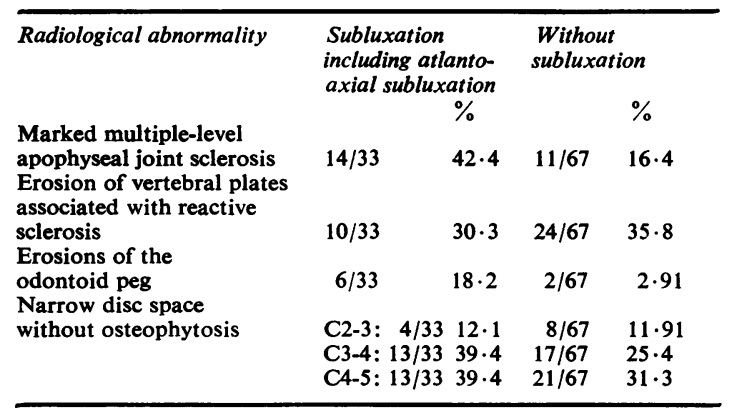

arthritis affecting the cervical spine. In particular, marked apophyseal joint sclerosis affecting several disc levels and erosions of the odontoid peg was found more frequently in the subluxation group than in the group without subluxation. As might be expected, disc space narrowing affecting the upper cervical disc levels was more frequent in the subluxation group (see Table 1).

Of the 67 patients who did not have subluxation 33 had osteoarthritic changes only with disc space narrowing of C5-6 and C6-7 levels with osteophytosis. Fifteen patients had normal serial radiographs, and the remaining 19 patients had less than 3 of the radiographic criteria given in Fig. 1.

\section{Discussion}

This prospective study has clarified the natural history of cervical spine changes in rheumatoid disease in several ways. Firstly, subluxation in the cervical spine may be an early complication. In this series $12 \%$ of patients developed atlanto-axial subluxation, a further $20 \%$ developed subaxial subluxation, while $3 \%$ developed vertical subluxation. Over $80 \%$ of these patients showed the first evidence of subluxation within 2 years of disease presentation, and all cases of subluxation were apparent within 5 years. Early cases of subluxation have previously been reported. Mathews ${ }^{10}$ and Meikle and Wilkinson ${ }^{11}$ found patients in whom atlanto-axial subluxation occurred within 2 years of disease presentation. It is not surprising, however, that subluxation may be apparent when the disease first presents, since erosions in the peripheral joints may also be detectable at an early stage.4 12

The $12 \%$ incidence of atlanto-axial subluxation in this series differs from previous studies. Meikle and Wilkinson ${ }^{11}$ found that atlanto-axial separation exceeded $2.5 \mathrm{~mm}$ in flexion in $37 \%$ of rheumatoid patients, while Stevens et al. ${ }^{13}$ found an incidence of $36 \%$ using $3 \mathrm{~mm}$ as the upper limit of normal. 
The mean age, however, in the present series was 47.6 years, whereas the mean age in the latter 2 studies was 53.8 years. It is highly likely, therefore, that with longer follow-up, more cases of atlantoaxial subluxation will become apparent. This gives support to the concept that the incidence of AAS is related to disease duration.

A proportion of patients with AAS, perhaps $40 \%$, do not demonstrate any deterioration in the degree of subluxation with respect to time. The reason may be that the erosive process has been controlled in this region. In addition, fibrosis or ankylosis at the vertebral end plate and apophyseal joint level may prevent any further subluxation. In this study erosions of the vertebral end plates were found with similar frequency in the subluxation and nonsubluxation group, so that damage to the apophyseal joints, by the rheumatoid process, may be more important than vertebral end-plate erosion in the mechanism of subluxation. The increase in stiffness of the cervical spine, a common symptom, may have a protective role in the presence of subluxation, by reducing spinal cord trauma or injury to the cord vasculature. It is important to screen those patients with highly active, erosive rheumatoid disease for cervical subluxation, because some of these patients, despite suppressive therapy, demonstrate a steady deterioration in their atlanto-axial subluxation without necessarily a deterioration in their symptoms. When these patients are $x$-rayed it may be useful to remember that the full degree of atlanto-axial instability may not be seen radiologically if the cervical spine flexion is markedly impaired. The comparison of serial films should therefore take into account the degree of cervical spine flexion.

For the anaesthetist the detection of subluxation is always important. It is mandatory to $x$-ray the cervical spine in flexion and extension in all patients with rheumatoid disease who may need a general anaesthetic, since unsuspected cases of subluxation may be found. This study has clearly shown that patients with early disease may demonstrate marked subluxation, and it is well known that many patients with atlanto-axial subluxation may have no symptoms referrable to the neck. ${ }^{13}$ On clinical examination marked limitation in neck mobility may give a clue that subluxation is present. It is also possible that $x$-rays of patients with marked limitation in neck movement may not demonstrate the true degree of subluxation which may be produced during anaesthesia when the neck muscles are relaxed and protective spasm is absent.

None of the patients in this series developed neurological signs, but the mean follow-up period was only 7 years 2 months. Sharp and Purser ${ }^{14}$ reported an incidence of cervical myelopathy in $23 \%$ of their patients, while Stevens et al. ${ }^{13}$ showed that twothirds of their patients with AAS showed evidence of myelopathy. Interestingly, myelopathy can be found in patients with rheumatoid arthritis who do not have subluxation, implying that other mechanisms may be important in the development of myelopathy. ${ }^{13}$ The mean age in the present series, however, was lower than in those 2 studies, suggesting that further follow-up may well reveal patients who develop neurological signs. This study suggests, however, that the neurological signs of cervical myelopathy do not occur in the early stages of the disease and that they are probably related to disease duration.

There is still much controversy about the association of atlanto-axial subluxation and parameters of disease, duration of disease, sex, and steroid therapy. Mathews ${ }^{10}$ found that atlanto-axial subluxation was related to disease duration, steroid therapy, and was more frequent in males. Conlon et al. ${ }^{15}$ did not find an association between atlanto-axial subluxation and the above features. In the present study atlanto-axial subluxation was more common in females (female to male ratio $3: 1$ ) which is in agreement with previous reports which have shown a female predominance. ${ }^{11} 13$ This study also confirms the finding that steroids exert their own deleterious effects in exacerbating the atlanto-axial changes. ${ }^{11} 16$

Subluxation was the most convincing evidence of rheumatoid involvement of the cervical spine. The next most useful features to correlate with subluxation were erosive damage to the odontoid peg and marked multiple-level apophyseal joint sclerosis, features which Rasker and $\operatorname{Cosh}^{16}$ found to be significantly associated with the American Rheumatism Association grading. Erosion of the vertebral plates associated with sclerosis and disc space narrowing without osteophytosis occurred with similar frequency in the subluxation and nonsubluxation group. A longer follow-up study may reveal further information about the progression of subluxation and the association between subuxation and the development of neurological signs.

We thank Drs A. C. Boyle, S. Mattingly, and D. L. Woolf for permission to study their patients.

The study has been generously supported by the Arthritis and Rheumatism Council.

\section{References}

1 Sharp J. Differential diagnosis of ankylosing spondylitis. Br Med J 1957; i: 975-8.

2 Bland $\mathbf{J} \mathbf{H}$, Davis $\mathbf{P} \mathbf{H}$, London $\mathbf{M}$ G. Rheumatoid arthritis of the cervical spine. Arch Intern Med 1963;112: 892-8.

3 Lawrence J S. Radiological cervical arthritis in populations. Ann Rheum Dis 1976; 35: 365-71.

4 Fleming A, Crown J M, Corbett M. Early rheumatoid disease 1. Onset. Ann Rheum Dis 1976; 35: 357-60. 
5 Ropes M W, Bennett G A, Cobb S, Jacox R, Jessar R A. Diagnostic criteria for rheumatoid arthritis, 1958 revision. Ann Rheum Dis 1959; 18: 49-51.

- Bland J H. Rheumatoid arthritis of the cervical spine. $J$ Rheumatol 1974; 1 : 319-42.

7 Smith P H, Benn R T, Sharp J. Natural history of cervical luxation. Ann Rheum Dis 1972; 31 : 431-9.

8 McGregor M. The significance of certain measurements of the skull in the diagnosis of basilar impression. $B r J$ Radiol 1948; 21 : 171-81.

- Mathews J A. Atlanto-axial subluxation in rheumatoid arthritis. A 5 year follow-up study. Ann Rheum Dis 1974; 33: 526-31.

10 Mathews J A. Atlanto-axial subluxation in rheumatoid arthritis. Ann Rheum Dis 1969; 28: 260-6.

11 Meikle J A K, Wilkinson M. Rheumatoid involvement of the cervical spine: radiological assessment. Ann Rheum Dis 1971; 30: 154-61.
12 Jacoby R K, Jayson M I V, Cosh J A. Onset, early stages and prognosis of rheumatoid arthritis. A clinical study of 100 patients with 11 year follow-up. $\mathrm{Br} \mathrm{Med} J 1973$; ii: $96-100$.

13 Stevens J C, Cartlidge N E F, Saunders M, Appleby A, Hall M, Shaw D A. Atlanto-axial subluxation and cervical myelopathy in rheumatoid arthritis. $Q J \mathrm{Med}$ 1971; 40: 391-408.

14 Sharp J, Purser D W. Spontaneous atlanto-axial dislocation in ankylosing spondylitis and rheumatoid arthritis. Ann Rheum Dis 1961; 20: 47-77.

15 Conlon P W, Isdale I C, Rose B S. Rheumatoid arthritis of the cervical spine. Ann Rheum Dis 1966; 25: 120-6.

16 Rasker J J, Cosh J A. Radiological study of cervical spine and hand in patients with rheumatoid arthritis of 15 years' duration: an assessment of the effects of corticosteroid treatment. Ann Rheum Dis 1978; 37: 529-35. 